\title{
Small Saturating Inductors for More Compact Switching Power Supplies
}

\author{
Luke Milner* Non-member \\ Gabriel A. Rincón-Mora* Non-member
}

\begin{abstract}
Other than by reducing power, extending battery life in portable microelectronics amounts to increasing power efficiency, which when coupled with accuracy, translates to increasing filter inductance. The problem with higher inductances is magnetic cores require more space to prevent the onset of saturation, so accuracy and efficiency (via their need for bulky inductors) hamper the miniaturization benefits gained from chip integration. This paper illustrates the time-domain and efficiency effects of inductor saturation in switched-inductor dc-dc converters and shows how they can accommodate saturation (with up to $65 \%$ reduction in inductance) with minimal impact on battery life and accuracy. Extending the useful range of an inductor in this fashion not only reduces PCB area and volume to a fraction (e.g., 30-50\%) of what an otherwise larger unsaturated inductor would require but also helps bridge the integration gap that enables practical system-on-chip (SoC) implementations.
\end{abstract}

Keywords : Inductors, Saturation, Switching Supplies, DC-DC Converters

\section{Small Switched-Inductor Power Supplies}

Switching supply circuits often demand more space than many emerging portable applications have available. Foregoing the supply circuit, however, is not an option because boosting or bucking a source voltage is typically a basic necessity, especially when $(i)$ drawing energy from high-energy but low-voltage sources like miniaturized fuel cells (1)-(3) and solar cells (4)-(5) and (ii) supplying energy-saving, dynamically adaptive systems (6)-(7). One challenge with building an efficient and accurate supply circuit, within the context of volume, is the need for large inductors because they transfer energy with minimal power losses and suppress voltage ripples in the output. Even in micropower systems, these components often double or triple the area and volume of the circuit (8)-(9). The fact that many state-of-the-art subsystems cannot tolerate the variance or voltage level of a single, common supply exacerbates the problem because several point-of-load (PoL) supply circuits (and their inductors) require even more space (10)-(11).

This paper proposes and verifies experimentally that switching supplies can accommodate smaller inductors by allowing their cores to saturate and therefore extending their useful power range while still requiring little space. By conforming switching converters into smaller spaces, emerging miniaturized platforms can now benefit from the high conversion efficiencies (i.e., extended battery-life performance) of switched-inductor circuits. Since small supplies facilitate integration, more converters can also fit into one unit, allowing a system to reap the efficiency and performance advantages of point-of-load regulation. Because the extent and consequences of inductor saturation in switching converters were unclear in prior literature, many state-of-the-art designs do not enjoy such improvements in battery life and regulation performance, limiting advances in biomedical implants, wireless microsensors, and other tiny applications. For those reasons, Sections II and III first describe how inductors saturate and why power-supply designers avoid saturation and Sections IV and $\mathrm{V}$ then illustrate and verify the actual time-domain and efficiency effects of saturation in switching power supplies. Section VI evaluates the results obtained and Section VII draws relevant conclusions.

\section{Inductor Saturation}

A switching dc-dc converter, be it an inverting or non-inverting buck, boost, or buck-boost circuit, transfers energy from an input source $V_{I N}$ to a load (i.e., an output $v_{O}$ ) by energizing (from $V_{I N}$ )

\footnotetext{
* Georgia Tech Analog, Power, \& Energy IC Lab
}

777 Atlantic Drive NW, Atlanta, GA, USA 30332 and de-energizing (to $v_{O}$ ) an inductor $L_{O}$ in alternate phases, as Fig. 1a generally depicts. More specifically, a constant energizing voltage $V_{E}$ across $L_{O}$ energizes $L_{O}$, increasing its current $i_{L}$ (where $i_{L}$ is $L_{O} \mathrm{~d} v_{L} / \mathrm{d} t$ ) and flux $\lambda_{L}$. Reversing the voltage across $L_{O}$ in the form of de-energizing voltage $V_{D E}$ releases $L_{O}$ 's energy and causes $i_{L}$ and $\lambda_{L}$ to decrease, but not along the initial energizing path, as Fig. $1 b$ shows.

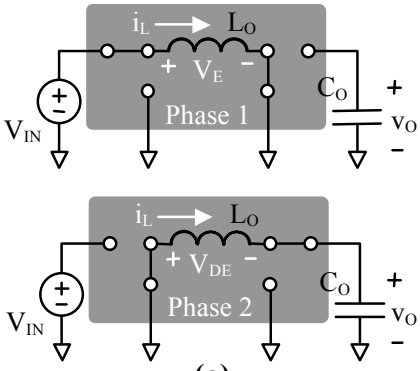

(a)

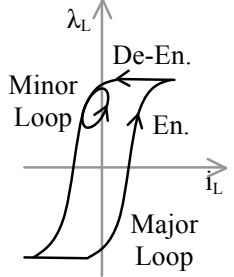

(b)
Fig. 1. (a) Energizing and de-energizing inductor $L_{O}$ (b) means flux increases and decreases along $L_{O}$ 's hysteresis loop.

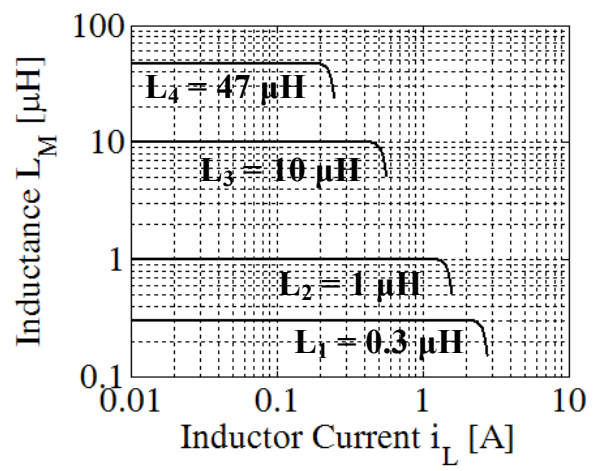

Fig. 2. Typical inductance-saturation graph (17).

As $L_{O}$ repeatedly energizes and de-energizes, $i_{L}$ and $\lambda_{L}$ traverse along a hysteresis loop, which means $L_{O}$ releases less energy than it stored in its energizing cycle (where the area in the loop represents the energy lost in the core (12)-(13)). (Note $L_{O}$ also loses power across the equivalent series resistance ESR of the copper coil.) Beyond a threshold current, the flux in the core begins to saturate, causing the range of $i_{L}$ (i.e., ripple current $\Delta i_{L}$ ) and the hysteresis loop to expand (13)-(16), which is to say both $L_{O}$ 's $\Delta i_{L}$ and core losses increase. Another way to describe this 
effect is through inductance because the increase in $\Delta i_{L}$ is equivalent to a decrease in $L_{O}$. Datasheets, for example, illustrate that inductance decreases at higher currents (17), as Fig. 2 shows, albeit with little insight into the actual time-domain operation and power-efficiency effects of saturation.

\section{Power-Loss Argument}

Switched-inductor circuits are ideally lossless because the power switches experience nearly negligible voltage (and therefore power) drops and $L_{O}$ and $C_{O}$ introduce next to no series resistances in the power path. In practice, however, the feedback controller draws quiescent current from the supply, the drivers lose energy in charging and discharging the parasitic gate capacitance of the switches, and $L_{O}$ 's average and ripple currents $i_{L(a v g)}$ and $\Delta i_{L}$ dissipate Ohmic conduction losses $P_{C}$ across the finite resistances that the switches, $L_{O}$, and $C_{O}$ actually present:

$$
P_{C}=R_{E Q 1} i_{L(a v g)}{ }^{2}+R_{E Q 2}\left(\frac{\Delta i_{L}{ }^{2}}{12}\right),
$$

where $R_{E Q 1}$ and $R_{E Q 2}$ are the combined stray resistances $L_{O}, C_{O}$, and the power switches introduce in $i_{L(a v g)}$ 's path through $L_{O}$ and $\Delta i_{L}$ 's path into $C_{O}$, respectively, and $\Delta i_{L}^{2} / 12$ is the root-mean-squared (RMS) value of $i_{L}$ 's ripple, which is triangular in nature (18). When considering the high efficiency objectives of the supply and the reduction of energy delivered to the output that saturating $L_{O}$ causes, limiting the sum of these losses is important. As a result, because magnetic core losses and $\Delta i_{L}$ (and its derivative losses across $R_{E Q 2}$ in $P_{C}$ ) increase when $L_{O}$ saturates, designers typically select the core's cross-sectional area and volume to be large enough to sustain (without saturating) the flux associated with the highest expected $i_{L}$. They further oversize $L_{O}$ to offset the fact that the saturation characteristics in datasheets are absent or poorly specified. The underlying aim of this paper is to show that such design practices are overly cautious, which is to say designs can survive the saturating effects of smaller inductors and, in consequence, reap the benefits of higher integration.

\section{Actual Saturated Response}

Representative Circuit: Because all dc-dc converters energize and de-energize inductors in the same way: by applying quasi-constant positive and negative voltages across a power inductor in alternating cycles across a switching period (19)-(23), the power inductor of all switching dc-dc converters similarly conduct dc and triangular ripple currents $i_{L(a v g)}$ and $\Delta i_{L}$. Therefore, the buck converter in Fig. 3 (and Fig. 4a), just like any other switching dc-dc supply, is a good means of verifying and demonstrating how $i_{L}$ in switched-inductor circuits generally responds to saturating conditions. The only differentiating factor between these switching circuits, from the perspective of $i_{L}$, is the magnitude of energizing and de-energizing voltages $V_{E}$ and $V_{D E}$. In the buck case, $L_{O}$ (from Fig. $4 \mathrm{~b}$ ) energizes from $V_{I N}$ to $v_{O}$ with PMOS power switch $M_{P}$ so $V_{E}$ equals $V_{I N}-v_{O}$, and de-energizes to $v_{O}$ from ground with NMOS power switch $M_{N}$ so $V_{D E}$ is $-v_{O}$. As in all switching converters, in addition to $L_{O}$ and its connecting switches $M_{P}$ and $M_{N}$, the supply circuit also includes output capacitor $C_{O}$, drivers, and a feedback controller, the last two of which (and in some cases, also $M_{P}$ and $M_{N}$ ) are often embedded in a common integrated circuit (IC).

Time-Domain Response: With respect to inductor saturation and power efficiency, what is important is $i_{L}{ }^{\prime} \mathrm{s} d \mathrm{dc}$ and ripple currents $i_{L(a v g)}$ and $\Delta i_{L}$, in other words, $i_{L}$ 's steady-state time-domain waveform. From this perspective, the dynamics of the control loop (e.g., feedback amplifier, etc.) are irrelevant. As a result, to decouple the effects of the controller from $L_{O}$ 's saturation effects on $i_{L(a v g)}$ and $\Delta i_{L}$, the foregoing discussion and measurements assume a pulse-width modulated (PWM) square-wave signal $v_{P W M}$ is available and ready to drive $L_{O}$ 's energizing and de-energizing switches in the power stage. That is to say, a function generator defines and sources the control signal in Fig. $3\left(v_{P W M}\right)$ that the dead-time block uses to produce the non-overlapping gate voltages that drive $M_{P}$ and $M_{N}$.

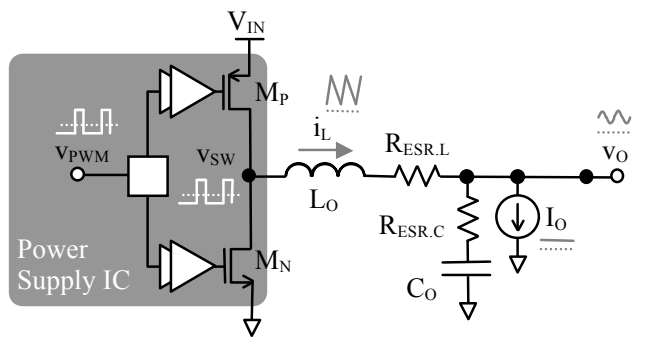

Fig. 3. Switching buck dc-dc converter.
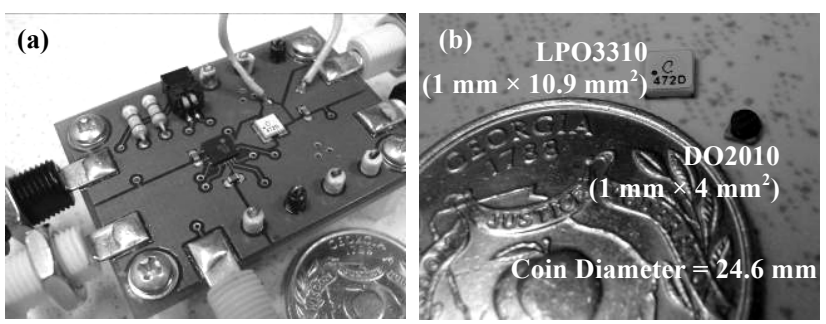

Fig. 4. (a) Switching converter printed circuit board (PCB) built to test (b) inductors LPO3310 and DO2010.

Because the circuit impresses time-invariant energizing and de-energizing voltages $V_{E}$ and $V_{D E}$ across $L_{O}$, instantaneous inductance $L_{M}$ determines $i_{L}$ 's rising and falling slopes, that is, $\mathrm{d} i_{L}{ }^{+} / \mathrm{d} t$ is $V_{E} / L_{M}$ and $\mathrm{d} i_{L}{ }^{-} / \mathrm{d} t$ is $V_{D E} / L_{M}$. As such, the effects of saturation manifest as changes in $i_{L}$ 's slope across time (24). Fig. 5 , for example, illustrates that the rising and falling slopes of $i_{L}$ in the buck converter of Fig. 3 are constant as long as $i_{L}$ remains below $2 \mathrm{~A}$. Once above this threshold, $L_{O}$ saturates and both rising and falling slopes increase, the degree of which $L_{O}$ 's effective inductance determines. Measuring this instantaneous inductance $L_{M}$ amounts to sampling $i_{L}$ (via a Hall-effect probe, for example) and its slope across time (with an oscilloscope). Fig. 5 therefore demonstrates that $L_{M}$ is constant when the core is unsaturated and decreases with increasing $i_{L}$ only when $L_{M}$ saturates, when $i_{L}$ surpasses $2 \mathrm{~A}$ in this case. In practical terms, as graphically shown, saturation increases current ripple $\Delta i_{L}$.

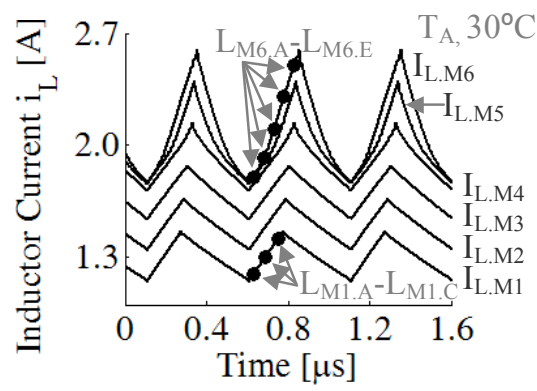

Fig. 5. Experimental inductor-current waveforms as a $1-\mu \mathrm{H}$ inductor saturates.

Inductance Variation: By repeatedly calculating inductance at several points on each waveform for increasing values of average inductor current $i_{L(\text { avg })}$ at various ambient temperatures, Fig. 6 maps (from experimental measurements) how $L_{M}$ changes across instantaneous and average $i_{L}$ values and temperature. These results show that $L_{M}$ decreases from a nominal non-saturated value $\left(L_{N S}\right)$ 
of $1.1 \mu \mathrm{H}$ to a constant and lower saturated value $\left(L_{S}\right)$ of $0.35 \mu \mathrm{H}$ when the core saturates, which represents a $65 \%$ reduction in $L_{M}$. The bimodal piecewise linear response of $i_{L}$ 's rising and falling slopes in the saturated waveforms of Fig. 5 graphically corroborate that $L_{M}$ drops to a constant $\left(L_{S}\right) . L_{M}$ does not decrease below $L_{S}$ because saturating the magnetic core is equivalent to replacing it with an air core the inductance of which is roughly $0.35 \mu \mathrm{H}$. Fig. 6, however, further reveals that the onset of saturation decreases with $i_{L(a v g)}$, which previously reported literature did not show (24)-(27). Increasing temperature (from 30 to $90{ }^{\circ} \mathrm{C}$, for example) also has similar effects on the onset of saturation; that is, higher temperatures induce a lower saturation threshold (14). The last two effects are not mutually exclusive, though, because higher $i_{L(a v g)}$ increases the power lost in $L_{M}$ as heat, so temperature also rises with $i_{L(a v g)}$. Nevertheless, the result is the same: the onset of saturation decreases with higher $i_{L(a v g)}$ and higher ambient temperatures.

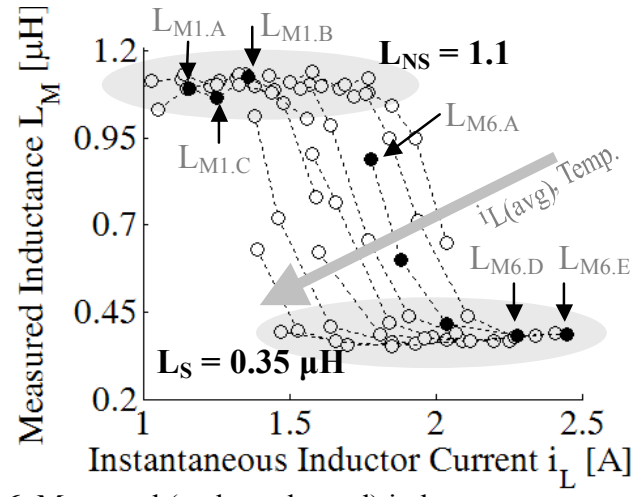

Fig. 6. Measured (and overlapped) inductances across current and temperature (at 30,60 , and $90^{\circ} \mathrm{C}$ ).

\section{Power Efficiency in Saturation}

The effect of saturation on efficiency is noticeable but often tolerable. To start, conduction losses $P_{C}$ increase with increasing current ripple $\Delta i_{L}$, but this only happens when $i_{L(a v g)}$ (i.e., output current $I_{O}$ ) is sufficiently high to induce saturation, and even then, $\Delta i_{L}$ only increases by a factor of 3 , given the $65 \%$ reduction in $L_{O}$ when the core saturates. Consider that $P_{C}$ (according to Eq. 1) is $771 \mathrm{~mW}$ when $R_{E Q}$ is $300 \mathrm{~m} \Omega, R_{E S R . C}$ is $50 \mathrm{~m} \Omega, i_{L(a v g)}$ is $1.6 \mathrm{~A}$, and $\Delta i_{L}$ is $350 \mathrm{~mA}$, and tripling $\Delta i_{L}$, as saturation would, increases $P_{C}$ by $2.7 \%$ to $792 \mathrm{~mW}$.

As stated earlier, core losses also increase with saturation, and this loss is the difference between $L_{O}$ 's stored and delivered energy in the alternating cycles of the converter. The area $\left(A_{H Y S}\right)$ enclosed by the minor $\lambda_{L}-i_{L}$ hysteresis loop that $i_{L}$ creates in Fig. 1 (as $i_{L}$ rises and falls each cycle) represents this loss (13), (28), which means small hysteresis loops indicate low core losses. Fig. 7 extracts this $\lambda_{L}-i_{L}$ information from Fig. 5 by extrapolating and integrating inductor voltage $v_{L}$ from the measured data (13), (29)-(30), where $v_{L}$ is the difference between energizing (or de-energizing) voltage $V_{E}$ (or $V_{D E}$ ) and the voltage across $L_{O}$ 's ESR, which is $i_{L} R_{E S R . L}$ (because Faraday's Law dictates that $v_{L}$ represents the rate of change of magnetic flux-linkage through the inductor's core). Therefore, accounting for the resistive voltage drop and integrating the remaining voltage is a way to calculate the flux that is plotted in Fig. 7.) Multiplying the energy loss by the converter's switching frequency $f_{S W}$ yields inductor-core power loss $P_{L . C O R E}$ :

$$
P_{L . C O R E}=f_{S W} \oint i_{L} \mathrm{~d} \lambda_{L}=f_{S W} A_{H Y S} .
$$

Experimental results in Fig. 7 show that the minor $\lambda_{L}-i_{L}$ hysteresis loop first shifts up and to the right with increasing $i_{L \text { (avg) }}$ (because $\lambda_{L}$ and $i_{L}$ increase) and then distorts and widens with $i_{L(a v g)}$ past saturation (as core losses and $\Delta i_{L}$ increase), which (31) corroborates. Before the core saturates, since $P_{L C O R E}$ (calculated by integrating the closed contours of Fig. 7) remains constant and $P_{C}$ rises with $i_{L(a v g)}$, the percentage of $P_{L . C O R E}$ in the total power lost in $L_{O}\left(P_{L}\right)$ decreases with $i_{L(\text { avg })}$ from $24 \%$, in this case, to $13 \%$ when $i_{L(a v g)}$ increases from 1.3 to $1.7 \mathrm{~A}$. (Note $P_{L}$ is the average of the product of $i_{L}$ and $v_{L}$, which is also the sum of conduction losses $R_{E S R . L} i_{L}^{2}$ and $P_{\text {L.CORE }}$ ). In saturation, $P_{L . C O R E}$ increases from 63 to $158 \mathrm{~mW}$, and from $13 \%$ of $P_{L}$ back to $23 \%$. In effect, saturation negates the decreasing impact of the core on efficiency as $i_{L(a v g)}$ increases, but only to a similar extent that decreasing $i_{L(a v g)}$ does when not saturated, which means saturation is not necessarily detrimental to the converter's efficiency performance.

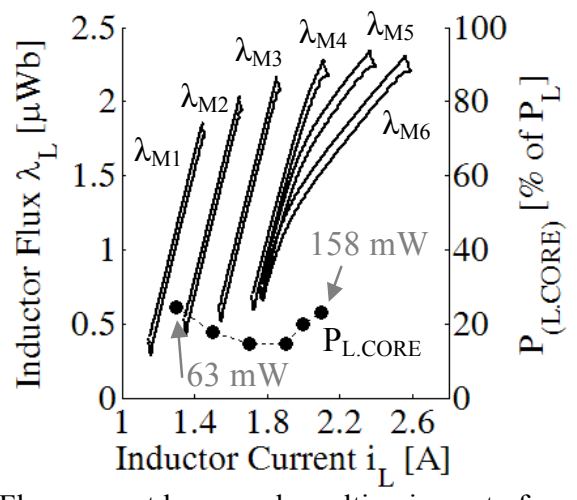

Fig. 7. Flux-current loops and resulting impact of core losses.

In the end, core saturation degrades efficiency, although only to a certain extent, and because current ripple $\Delta i_{L}$ increases, output voltage ripple $\Delta v_{O}$ also increases, which means saturation also degrades converter accuracy. The converter remains functional in saturation, however, efficiently regulating $v_{\mathrm{O}}$ in spite of slow and sudden changes in line voltage and load. Efficiency and accuracy are therefore legitimate trade-offs for volume, especially since the propensity for large currents and saturation in portable applications is low.

\section{Discussion}

Ultimately, the benefit of reducing inductance is smaller volume. The inductor used in the above experiments, for instance, which was a $3.3 \times 3.3 \times 1.0 \mathrm{~mm}^{3}\left(10.9 \mathrm{~mm}^{3}\right)$ LPO3310 with a ferrite core (similar to the on-package inductor used in (32)), a nominal inductance of $1.0 \mu \mathrm{H}$, and a nominal saturation limit of 1.6 A, was still effective past its saturation point to $2.1 \mathrm{~A}$. The actual (practical) limit, which exceeds $2.1 \mathrm{~A}$, is when the inductor overheats, which depends on several factors like printed-circuit-board (PCB) layout, inductor package, and duration of large loads. For reference, consider that among inductors that do not saturate at $2 \mathrm{~A}$, the smallest (as inferred from (17)) is $20 \%$ larger at $12.4 \mathrm{~mm}^{3}$, despite its smaller footprint. Similarly, a DO2010 behaves like an LPO3310 up to at least $2 \mathrm{~A}$, and the smallest inductor with a 2-A rating (ME3215) requires twice the area and more than 3 times the volume; in other words, a DO2010 yields $50 \%$ and $67 \%$ drop in area and volume, respectively. This reduction is of paramount importance because the inductor typically dominates the area and volume of most converter circuits (8)-(9), (32). Of course, these savings, as Table 1 summarizes, vary with manufacturer and technology, and the inductors considered here for proof of concept may not be optimal for a given application.

Table 1. Comparison of Inductor Size and Performance 


\begin{tabular}{||c|c|c|c||}
\hline & LPO3310 & DO2010 & ME3215 \\
\hline \hline $\mathbf{L}_{\mathbf{O}}$ & $1.0 \mu \mathrm{H}$ & $1.0 \mu \mathrm{H}$ & $1.0 \mu \mathrm{H}$ \\
\hline $\mathbf{R}_{\mathbf{D C}}$ & $76 \mathrm{~m} \Omega$ & $200 \mathrm{~m} \Omega$ & $58 \mathrm{~m} \Omega$ \\
\hline Area & $10.9 \mathrm{~mm}^{2}$ & $4.0 \mathrm{~mm}^{2}$ & $8.0 \mathrm{~mm}^{2}$ \\
\hline Volume & $10.9 \mathrm{~mm}^{3}$ & $4.0 \mathrm{~mm}^{3}$ & $12.0 \mathrm{~mm}^{3}$ \\
\hline I $_{\text {RATED }}$ & $1.6 \mathrm{~A}$ & $1.8 \mathrm{~A}$ & $2.3 \mathrm{~A}$ \\
\hline I $_{\text {EXP. }}{ }^{*}$ & $2.1 \mathrm{~A}$ & $2.1 \mathrm{~A}$ & \\
\hline
\end{tabular}

While high efficiency is desirable at all load levels, efficiency at light-to-moderate loads in portable applications is vital and more important than at peak loads. Wireless CDMA handsets, for instance, normally idle at low-to-moderate power levels and consume peak power only a fraction of the time, which is why their peak-to-average-power ratios (PAPR) are high (33). In other words, idling consumes most of the energy stored in the battery so light- (not peak-) load efficiency ultimately determines operational life (34). Many wireless microsensor applications exhibit even larger PAPRs because data collection and transmission is less frequent. What all this means is that trading some peak-power efficiency for reductions in PCB real estate (by using smaller inductors) is justifiable and optimal for many portable applications.

Saturation also degrades converter accuracy because a higher inductor ripple current $\Delta i_{L}$ induces a larger ripple voltage across $C_{O}\left(\Delta v_{O}\right)$, increasing the systematic noise content in the output of the converter. In the presented converter, because switching frequency $f_{S W}$ was constant at $2 \mathrm{MHz}$, saturation tripled $\Delta i_{L}$, inducing $\Delta v_{O}$ to increase by a corresponding percentage (e.g. from $3.2 \mathrm{mV}$ to $8.5 \mathrm{mV}$ when $C_{O}$ and $R_{E S R . C}$ are $10 \mu \mathrm{F}$ and $20 \mathrm{~m} \Omega$, respectively). While this degradation in accuracy is important, it may not be as critical in systems where linear, low-dropout regulators buffer (and filter) the converter to supply power to sensitive analog blocks (18), reducing the ripple by 30-40 dB. In the case converter accuracy is nonetheless important, modifying the loop's control strategy to regulate $i_{L}$ 's ripple $\Delta i_{L}$ by modulating $f_{S W}$, as in hysteretic converters whose response to load dumps is substantially faster than competing schemes (35), circumvents this accuracy degradation, albeit at the expense of a slightly variable $f_{S W}$.

\section{Conclusions}

This paper demonstrates that a $3.3 \times 3.3 \times 1.0-\mathrm{mm}^{3}\left(10.9-\mathrm{mm}^{3}\right)$ ferrite-core $1.0-\mu \mathrm{H}$ inductor rated for $1.6 \mathrm{~A}$ operates well in switching dc-dc converters past its saturation point to $2.1 \mathrm{~A}$, and that saturating inductors can reduce PCB area and space by $50 \%$ and $67 \%$, respectively, over competing alternatives, while increasing total peak-power conduction losses by $2.7 \%$ from 771 to $792 \mathrm{~mW}$. Experimental results further show that inductance decreases from $1 \mu \mathrm{H}$ to no less than $0.35 \mu \mathrm{H}$, and that the onset of saturation decreases with higher average currents and ambient temperatures, which previous studies did not show. While a few percentage-points increase in peak-power efficiency is appreciable, its impact on battery life can be, for the most part, negligible in mobile devices because they tend to idle most of the time, which means light- (not peak-) load efficiency is critical. The point is that using a smaller inductor may save as much real estate as the converter IC (itself) requires, and the savings multiply in point-of-load $(\mathrm{PoL})$ architectures where several inductors may be necessary. Freeing space in this way might even enable subsystems to adopt dynamic scaling schemes (6)-(7), which increase power efficiency when and where it matters most. The benefits of extending the operational limits of an inductor are even more prominent in system-on-chip (SoC) solutions because using above-the-die (in-package) magnetic cores, which exhibit low saturation thresholds, eliminates the PCB real estate that off-chip inductors would otherwise demand.

\section{References}

(1) L. Palma, et. al, "Design Considerations for a Fuel Cell Powered DC-DC Converter for Portable Applications," $21^{\text {st }}$ Annual IEEE Applied Power Electronics Conference and Exposition, March 19-23, 2006, pp. 1263-1268.

(2) M. Chen, et. al, "Design Methodology of a Hybrid Micro-Scale Fuel Cell-Thin-Film Lithium Ion Source," $50^{\text {th }}$ Midwest Symposium on Circuits and Systems, August 5-8, 2007, pp. 674677.

(3) L. Palma, M.H. Todorovic, and P.N. Enjeti, "Analysis of Common-Mode Voltage in Utility-Interactive Fuel Cell Power Conditioners," IEEE Transactions on Industrial Electronics, Vol. 56, No. 1, January 2009, pp. 20-27.

(4) M.J. Vazquez, J.M.A. Marquez, and F.S. Manzano, “A Methodology for Optimizing Stand-Alone PV-System Size Using Parallel-Connected DC/DC Converters," IEEE Transactions on Industrial Electronics, Vol. 55, No. 7, July 2008, pp. 2664-2673.

(5) H. Matsuo and F. Kurokawa, "New Solar Cell Power Supply System Using a Boost Type Bidrectional DC-DC Converter," IEEE Transactions on Industrial Electronics, Vol. IE-31, No. 1, February 1984, pp. 51-55.

(6) T. Kim, "Application-Driven Low-Power Techniques Using Dynamic Voltage Scaling," Proc. 12th IEEE International Conference on Embedded and Real-Time Computing Systems and Applications, August 16-18, 2006, pp. 199-206.

(7) L. Yuan and G. Qu, "Analysis of Energy Reduction on Dynamic Voltage Scaling-Enabled Systems," IEEE Transactions on Computer-Aided Design of Integrated Circuits and Systems, Vol. 24, No. 12, December 2005, pp. $1827-1837$.

(8) S. Musunuri and P.L. Chapman, "Optimization Issues for Full-Integrated CMOS DC-DC Converters," $37^{\text {th }}$ Industry Applications Conference, Vol. 4, October 2002, pp. 2405-2410.

(9) N. Wakou, et al., "Micro Power Supply Using Thin Film Magnetic Core," International Magnetics Conference, April 2003, p. CP-01

(10) N. Pham, et. al, "Design Methodology for Multiple Domain Power Distribution Systems," Proc. of $54^{\text {th }}$ Electronic Components and Technology Conference, Vol. 1, June 1-4, 2004, pp. 542-549.

(11) S. Deuty, "Exploring the Options for Distributed and Point of Load Power in Telecomm and Network Applications," $26^{\text {th }}$ Annual International Telecommunications Energy Conference, September 19-23, 2005, pp. 223-229.

(12) S. Iyasu, et. al, "A Novel Iron Loss Calculation Method on Power Converters Based on Dynamic Minor Loop," European Conference on Power Electronics and Applications, September 11-14, 2005, pp. 9.

(13) T. Shimizu, et al., "Mitigation of Inductor Loss Based on Minor-Loop Hysteresis Characteristics," 25th International Telecommunications Energy Conference, October 2003, pp. 834-839.

(14) P.R. Wilson, J.N. Ross, and A.D. Brown, "Simulation of Magnetic Component Models in Electric Circuits Including Dynamic Thermal Effects," IEEE Transactions on Power Electronics, Vol. 17, January 2002, pp. 55-65.

(15) R. Salas, J. Pleite, E. Olias, and A. Barrado, "Nonlinear Saturation Modelling of Magnetic Components for Circuit Simulation," IEEE International Magnetics Conference, May 2006, p. 993.

(16) J.B. Wang, R. Li, and J. Chen, "Efficiency Comparison of Full Bridge Converters in Considered Magnetic Saturation," IEEE Industrial Electronics Conference, November 2008, pp. 717-722.

(17) Coilcraft Datasheets for LPO3310, ME3215, and DO2010, http://www.coilcraft.com/smpower.cfm.

(18) G.A. Rincón-Mora, Power Management ICs - A Top-Down Design Approach, Lulu, 2005.

(19) E.T. Moore and T.G. Wilson, "Basic Considerations for DC to DC Conversion Networks," IEEE Transactions on Magnetics, Vol. 2, No. 3, September 1966, pp. 620-624.

(20) R. Severns and G. Bloom, Modern DC-to-DC Switchmode Power Converter Circuits, Van Nostrand Reinhold, 1985.

(21) S. Ćuk, "General Topological Properties of Switching Converters," IEEE Power Electronics Specialists Conference, 1979, pp. 109-130.

(22) K.H. Liu and F.C. Lee, "Topological Constraints on Basic PWM Converters," IEEE Power Electronics Specialists Conference, 1988, pp. 164-172.

(23) R. Erikson, "Synthesis of Switched-Mode Converters," IEEE Power Electronics Specialists Conference, 1983. pp. 9-22.

(24) D.H. Boteler, "Characteristics of Time-Varying Inductance," IEEE Transactions on Magnetics, Vol. 30, No. 2, March 1994, pp. 172-176.

(25) E. Sugawara, et al., "Micro Inductor For Flip Chip Micro Power Source," International Magnetics Conference, April 2003, p. EA-05.

(26) E.J. Brandon, et al., "Fabrication and Characterization of Microinductors for Distributed Power Converters, IEEE Transactions on Magnetics, Vol. 39, No. 4, July 2003, pp. 2049-2056.

(27) B. Orlando, et al., "Low-Resistance Integrated Toroidal Inductor for Power Management," IEEE Transactions on Magnetics, Vol. 42, No. 10, October 2006, pp. 3374-3376

(28) J.S. Lipowski, "Analytical Models of Magnetic Saturation and Hysteresis in a Nonlinear Inductance For Application in Simulation Studies," International Jourunal for Computation and Mathematics in Electrical and Electronic Engineering, Vol. 14, No. 2, September 1985, pp. 187-201. 
(29) P.L. Chapman and S.D. Sudhoff, "Dynamic Lossy Inductor Model for Power Converter Simulation," Applied Power Electronics Conference, March 2002, pp. 137-143.

(30) S. Lizón-Martínez et al., "Measurement of Asymmetric Minor Loops in Soft Ferrites Up to Medium Frequencies," IEEE Instrumentation and Measurement Technology Conference, May 2007, pp. 1-4.

(31) S. Iyasu, et. al, "A Novel Iron Loss Calculation Method on Power Converters Based on Dynamic Minor Loop," European Conference on Power Electronics and Applications, September 11-14, 2005, pp. 9

(32) Z. Hayashi, et al., "High-Efficiency DC-DC Converter Chip Size Module With Integrated Soft Ferrite," IEEE Transactions on Magnetics, Vol. 39, No. 5, September 2003, pp. 3068-3072.

(33) J.B. Groe and L.E. Larson, CDMA Mobile Radio Design, Artech House, 2000.

(34) B. Sahu and G. A. Rincón-Mora, "A High-Efficiency Linear RF Power Amplifier with a Power-Tracking Dynamically Adaptive Buck-Boost Supply," IEEE Transactions on Microwave Theory and Techniques, Vol. 52, No. 1, January 2004, pp. 112-120.

(35) N. Keskar and G.A. Rincón-Mora, "A Fast, Sigma-Delta Boost DC-DC Converter Tolerant to Wide LC Filter Variations," IEEE Transactions on Circuits and Systems II, Vol. 55, No. 2, February 2008, pp. 198-202.

Luke Milner (Non-member) received a B.S. and an M.S.E.E in 2004

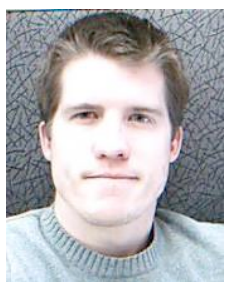
and 2006, respectively, from Georgia Tech, where he is currently pursuing a Ph.D. as a graduate research assistant in the Georgia Tech Analog, Power, \& Energy IC Lab. In 2005, he worked in the Power Technology Group at NSC in Santa Clara, $\mathrm{CA}$, and his research continues to focus on integrated dc-dc converters for portable electronics.

Gabriel A. Rincón-Mora (Non-member) (B.S., M.S., Ph.D., IET

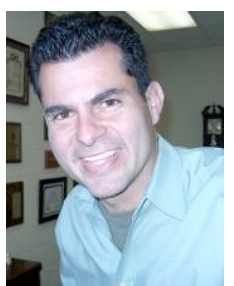

Fellow) worked for Texas Instruments in '94-'03, was appointed Adjunct Professor for Georgia Tech in '99-'01, and became a full-time faculty member at Georgia Tech in '01. His scholarly products include 8 books, 1 book chapter, over 125 scientific publications, 27 patents, over 26 commercial power management chip designs, and over 55 international speaking engagements. He received the "National Hispanic in Technology Award," "Charles E. Perry Visionary Award," a "Commendation Certificate" from the Lieutenant Governor of California, IEEE CASS Service Award, and Robins Air Force Base's "Orgullo Hispano" and "Hispanic Heritage" awards. He was inducted into Georgia Tech's "Council of Outstanding Young Engineering Alumni," elected IEEE CASS Distinguished Lecturer for '09-'10, and featured on the cover of Hispanic Business magazine as one of "The 100 Most Influential Hispanics." He has been Associate Editor for IEEE's TCAS II for '08-'11; Editorial Board Member for JOLPE since 2009; Chairman of Atlanta's IEEE SSCS-CASS Chapter since 2005; is Technical Committee Member for IEEE's CASS ASP and PECAS; Steering Committee Member for IEEE's MWSCAS; was General Chair for SRC's Energy and Power ICs Workshop in '09; Circuit Design Vice Chair for IEEE's '08 ICCDCS; Technical Program Chair for IEEE's '07 MWSCAS-NEWCAS; Technical Program Co-Chair for IEEE's '06 MWSCAS; Vice Chairman of Atlanta's SSCS-CASS Chapter in '04; and SBIR Selection Committee Review Panelist for NSF in '03-'07. 